\title{
Laboratory findings in predicting intensive care need and death of COVID-19 patients
}

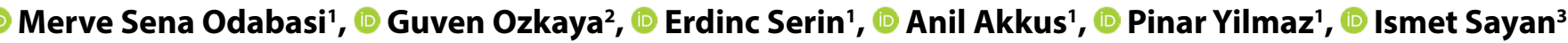 \\ 'Department of Biochemistry, Ministry of Health Istanbul Sisli Hamidiye Etfal Training and Research Hospital, Istanbul, Turkey \\ ${ }^{2}$ Department of Biostatistic, Uludag University Faculty of Medicine, Bursa, Turkey \\ ${ }^{3}$ Department of Anesthesia and Reanimation, Ministry of Health Istanbul Sisli Hamidiye Etfal Training and Research Hospital, Istanbul, Turkey
}

\begin{abstract}
Objectives: The ability to predict the course of COVID-19 is very valuable in terms of the optimal use of health resources. The aim of this study was to examine the value of biochemical and hematological parameters in the estimation of hospital stay, disease severity, and likelihood of death.

Methods: Routine blood analysis data of confirmed COVID-19 cases $(n=222)$ were collected and analyzed. The patients were divided into 3 groups: outpatient, inpatient, and patients requiring intensive care.

Results: There were significant differences between the 3 groups in terms of age, lymphocyte, neutrophil, hemoglobin, hematocrit, mean corpuscular volume (MCV), red blood cell distribution width (RDW), neutrophil-to-lymphocyte ratio (NLR), neutrophil-to-monocyte ratio (NMR), platelet-to-lymphocyte ratio (PLR), procalcitonin, C-reactive protein (CRP), and D-dimer values. Univariate analysis for mortality revealed significant differences in neutrophil, NLR, PLR, NMR, procalcitonin, and CRP values. Multivariable logistic regression yielded significant differences in only NMR and procalcitonin values. A positive correlation was determined between the length of hospital stay and age, MPV, procalcitonin, and D-dimer values.

Conclusion: The neutrophil count was the most appropriate parameter to predict the need for intensive care (area under the curve: 0.782 , sensitivity: $73 \%$, specificity: $75 \%$, with a cutoff of 4.43 ). The NMR and procalcitonin values were significant to predict death in multivariate analysis. Age, CRP, and D-dimer values were the parameters most associated with the duration of hospitalization.
\end{abstract}

Keywords: COVID-19, death, hemogram, intensive care unit, neutrophil

$\mathrm{T}^{\mathrm{s}}$ he Coronavirus 2019 (COVID-19) outbreak began in December 2019 in Wuhan, China. Despite efforts to contain it, the epidemic spread around the world. On March 11, 2020, the World Health Organization (WHO) confirmed a pandemic. The number of coronavirus cases had reached 65 million and the number of deaths attributed to the disease was 1.5 million worldwide in December 2020 [1].

Coronaviruses are an infectious agent for the common cold with subgroups that differ in contagiousness and risk of death. Severe acute respiratory syndrome coronavirus 2 (SARS-CoV-2), the virus that causes the illness coronavirus
2019 (COVID-19), is 10-20 times more transmissible than the original SARS-CoV $[2,3]$. Countries across the globe are struggling to cope with economic difficulties caused by quarantine measures, as well as health resource constraints, such as insufficient medical facilities and healthcare personnel. Clinical and laboratory findings that can provide a reliable COVID-19 prognosis will help to perform risk stratification to distinguish patients at high risk of developing serious disease. It will also provide guidance for the best possible management of health resources [4]. The identification of laboratory parameters that can be used to predict the severity or

Address for correspondence: Merve Sena Odabasi, MD. Department of Biochemistry, Ministry of Health Istanbul Sisli Hamidiye Etfal Training and Research Hospital, Istanbul, Turkey

Phone: +90 5382569380 E-mail: msdur88@gmail.com ORCID: 0000-0003-3774-4241

Submitted Date: February 02, 2021 Accepted Date: February 24, 2021 Available Online Date: March 22, 2021

${ }^{\circ}$ Copyright 2021 by International Journal of Medical Biochemistry - Available online at www.internationalbiochemistry.com OPEN ACCESS This work is licensed under a Creative Commons Attribution-NonCommercial 4.0 International License. 
the mortality risk will provide enhanced clinical situational awareness and facilitate appropriate treatment planning. Greater ability to manage care will reduce the disruption in the health system [5].

Abnormal hematology and biochemistry parameters can be used to diagnose infection-related tissue and organ damage and categorize patients at greater risk of developing severe disease [6]. They can also be used to recognize patients with a high probability of a poor prognosis and in monitoring the course of the disease. Studies have found that several parameters, such as white blood cell count, lymphocyte count, platelet count, and levels of interleukin-6, serum ferritin, and procalcitonin, can help predict the severity of COVID-19 $[7,8]$. The objective of this study was to examine the use of biochemical and hematological parameter data of hospitalization, disease severity, and mortality to predict disease progression in 222 patients with a positive polymerase chain reaction (PCR) test result for SARS-CoV-2.

The Turkish Ministry of Health COVID-19 adult patient treatment guideline specifies the criteria for hospitalization criteria for admission to the intensive care unit and intensive care treatment: dyspnea and respiratory distress, respiratory rate $\geq 30$ /minute, partial pressure of arterial oxygen $\left(\mathrm{PaO}_{2}\right)$ to fraction of inspired oxygen ratio $<300 \mathrm{mmHg}$, increased need for oxygen during monitoring, oxygen saturation $\left(\mathrm{SpO}_{2}\right)<90 \%$ or $\mathrm{PaO}_{2}<70 \mathrm{mmHg}$ despite $5 \mathrm{~L} /$ minute oxygen therapy, hypotension (systolic blood pressure [SBP] $<90 \mathrm{mmHg}$, drop of $>40 \mathrm{mmHg}$ from normal SBP, and mean arterial pressure $<65 \mathrm{mmHg}$ ), tachycardia >100/minute, acute kidney damage, acute liver function test disorder, signs of acute organ dysfunction such as confusion, acute bleeding diathesis, and patients with immunosuppression, troponin elevation and arrhythmia, lactate $>2 \mathrm{mmol}$, or the presence of skin disorders such as cutis marmaratus. The decision to stay in the ICU is made by the intensive care medical officer. The criteria for hospitalization are mild-moderate pneumonia with a respiratory rate $\geq 24 /$ minute and $\mathrm{SpO}_{2} \leq 93 \%$, mild-moderate pneumonia and blood values showing poor prognosis (blood lymphocyte count $<800 / \mu \mathrm{l}$, serum $\mathrm{C}$-reactive protein $[C R P]>10 \mathrm{x}$ normal upper limit, ferritin $>500 \mathrm{ng} / \mathrm{mL}$, D-dimer $>1000 \mathrm{ng} / \mathrm{mL}$, etc.), severe pneumonia (change in consciousness, respiratory distress, respiratory rate $\geq 30$ /minute, $\mathrm{SpO}_{2}<90 \%$ in room air, lung imaging of bilateral diffuse [ $>50 \%]$ involvement), hypotension $(<90 / 60 \mathrm{mmHg}$, mean blood pressure $<65 \mathrm{mmHg})$, tachycardia (>100/minute), sepsis, septic shock, myocarditis, acute coronary syndrome, arrhythmia, or acute kidney damage [9].

\section{Materials and Methods}

This study was approved by the Health Sciences University Sisli Hamidiye Etfal Training and Research Hospital Clinical Research Ethics Committee on September 22, 2020 (no: 3002). The need for written, informed consent was waived by the hospital ethics committee due to pandemic.

\section{Patients and data collection}

A total of 222 patients who were admitted to Sisli Hamidiye Etfal Training and Research Hospital between March 15, 2020 and June 15, 2020 with a positive COVID-19 PCR test and were not pregnant were included in the study. Routine biochemical and complete blood count parameters of COVID-19 patients were studied using a Beckman Coulter AU680 chemistry analyzer (Beckman Coulter, Inc., Brea, CA, USA) and a Mindray BC 6800 hematology analyzer (Mindray Medical International Co. Ltd., Shenzhen, China). Patient information and laboratory results were obtained retrospectively from the hospital and laboratory information management systems. The enrolled patients were divided into 3 groups: outpatients, inpatients, or patients needing intensive care. The hematological and biochemical data of the patients who received outpatient or inpatient treatment were recorded at the time of admission to the hospital. Data recorded on the first day in the ICU were used for patients who received intensive care.

\section{Statistical analysis}

The Shapiro-Wilk test was applied to determine the normality of distribution. The results were presented as mean \pm SD or median (minimum-maximum) for continuous variables. Categorical variables were described as frequency and percentage. Normally distributed data were compared with an independent samples t-test or one-way analysis of variance. The Kruskal-Wallis and Mann-Whitney $U$ tests were used for nonnormally distributed data. The Bonferroni test was used for multiple comparisons. Categorical variables were compared between groups using Pearson's chi-squared test and Fisher's exact test. Correlations between variables were tested using the Spearman correlation coefficient. Univariate logistic regression analysis was performed to assess the association between variables and disease progression. The odds ratio (OR) confidence intervals (Cls) were calculated at 95\%. Multivariate backward stepwise logistic regression analysis provided a second estimate of the OR after adjustment for confounding variables. Receiver operating characteristic (ROC) curve analysis of the statistically significant variables after binary logistic regression analysis was performed, and the area under curve (AUC) was calculated to evaluate the sensitivity and specificity of each variable/model to predict the severity of COVID-19. Cox proportional hazards regression model (forward step likelihood ratio approach) was used to perform multifactor analysis and calculate the hazard ratio (HR) values and $95 \% \mathrm{Cls}$ of the risk factors that were chosen on the basis of likely and relevant confounders after univariate analysis. The level of statistical significance was $\alpha=0.05$. The statistical analyses were performed with IBM SPSS Statistics for Windows, Version 23.0 (IBM Corp., Armonk, NY, USA).

\section{Results}

The median age of the patients was 56 years (range: 15-97 years) and $49.5 \%$ were men. Of the 222 patients, 138 had no 
Table 1. Demographic data of all of the study patients

\begin{tabular}{|c|c|c|c|c|c|}
\hline & $\begin{array}{l}\text { All patients } \\
(n=222)\end{array}$ & $\begin{array}{l}\text { Outpatient } \\
(n=74)\end{array}$ & $\begin{array}{l}\text { Inpatient } \\
(n=111)\end{array}$ & $\begin{array}{l}\text { ICU } \\
(n=37)\end{array}$ & $\mathbf{p}$ \\
\hline Age (years) & $56(15-97)$ & $38.5(15-87)^{a}$ & $60(25-97)^{b}$ & $62(46-93)^{b}$ & $<0.001$ \\
\hline Any comorbidity & $84(\% 37.84)$ & $0(\% 0)^{a}$ & $61(\% 54.95)^{\mathrm{b}}$ & $23(\% 62.16)^{b}$ & $<0.001$ \\
\hline Diabetes & $29(\% 13.06)$ & $0(\% 0)^{a}$ & $24(\% 21.62)^{\mathrm{b}}$ & $5(\% 13.51)^{b}$ & $<0.001$ \\
\hline Chronic obstructive pulmonary disease & $9(\% 4.05)$ & $0(\% 0)^{a}$ & $6(\% 5.41)^{a b}$ & $3(\% 8.11)^{b}$ & 0.043 \\
\hline Malignancy & $2(\% 0.90)$ & $0(\% 0)$ & $2(\% 1.80)$ & $0(\% 0)$ & - \\
\hline Chronic liver disease & $1(\% 0.45)$ & $0(\% 0)$ & $1(\% 0.90)$ & $0(\% 0)$ & - \\
\hline Exitus & $19(\% 8.56)$ & $0(\% 0)^{a}$ & $0(\% 0)^{a}$ & $19(\% 51.35)^{b}$ & $<0.001$ \\
\hline
\end{tabular}

Descriptive statistics are presented as median (minimum-maximum) or frequency with percentage. ICU: Intensive care unit.

comorbidities; $54 \%$ of the inpatients and $62 \%$ of the ICU patients had additional comorbid diseases. Diabetes mellitus (23\%) and hypertension (13\%) were the most common comorbidities. There were significant differences between the groups in age and the rates of comorbidities, diabetes, hypertension, cardiovascular disease, and chronic obstructive pulmonary disease. A total of 26 patients were transferred from inpatient clinics to the ICU and 11 patients were treated in the ICU upon presentation. In all, $51 \%$ of the ICU patients died while they were in hospital. There was a significant difference in the mortality rate between groups. The demographic data of the study group is shown in Table 1.

Biochemical and hematological data from the time of admission to the hospital were analyzed (Table 2). CRP, D-dimer, and procalcitonin parameters that were thought to be related to the clinical course were also analyzed to determine any association with hematological parameters. Significant differences between the 3 groups (outpatient, inpatient, ICU) were observed in age as well as lymphocyte, neutrophil, hemoglobin, hematocrit, mean corpuscular volume (MCV), red blood cell distribution width (RDW), neutrophil-to-lymphocyte ratio (NLR), neutrophil-to-monocyte ratio (NMR), platelet-to-lymphocyte ratio (PLR), procalcitonin, CRP, and D-dimer values. Pairwise comparisons revealed that there were significant differences in the NLR, procalcitonin, and D-dimer values of the 3 groups.

The relationship between parameters and admission to the ICU was analyzed. There was a significant difference in terms of age $(p=0.001)$ or gender $(p=0.025)$ between those with and without the need for intensive care. Data adjusted for age and gender were also evaluated using univariate analysis; the hemoglobin and hematocrit levels were low, while the neutrophil, mean platelet volume (MPV), platelet distribution width (PDW), MCV, NLR, PLR, NMR, procalcitonin, CRP, and D-dimer values were high. Multivariate analysis of intensive care need indicated a significant difference in age, MPV, and NMR values (Table 3).
ROC analysis of the parameters was performed to predict the need for intensive care treatment (Table 4). The parameter with the highest AUC was the neutrophil count, with a $73 \%$ sensitivity and a $75 \%$ specificity for a cutoff level of 4.43 (Fig. 1). The AUC was 0.77 for NLR (sensitivity: $71 \%$, specificity: $79 \%$ ) (Fig. 2) and the AUC was 0.678 for the NMR of 17.6 (sensitivity: $43 \%$, specificity: $89 \%$ ) (Fig. 3). In a logistic regression model of MPV and NMR, the AUC was lower than that of the neutrophil count.

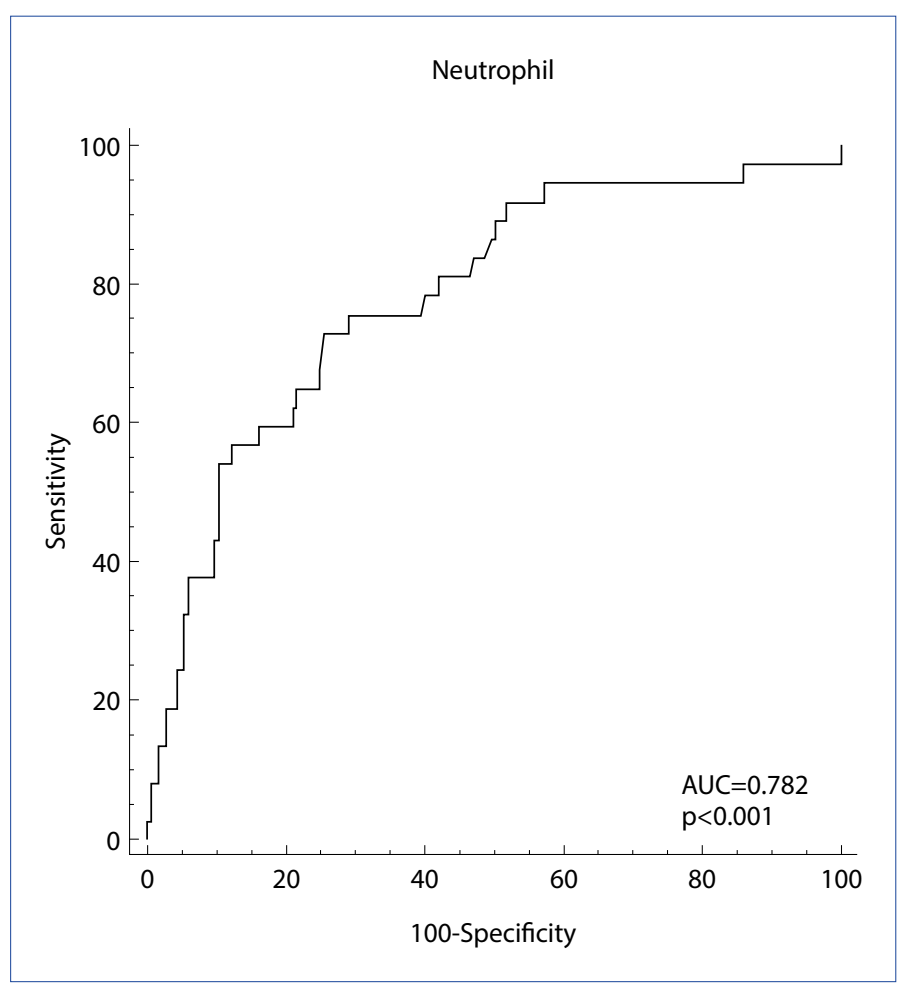

Figure 1. Receiver operating characteristic curve analysis for the neutrophil count as a predictor of the need for intensive care unit hospitalization.

AUC: Area under the curve. 
Table 2. Biochemical and hematological data of the patients

\begin{tabular}{|c|c|c|c|c|c|}
\hline & Outpatient $(n=74)$ & Inpatient ( $n=111)$ & ICU (n=37) & Total $(n=222)$ & $\mathbf{p}$ \\
\hline Neutrophil $\left(\times 10^{3} / \mu \mathrm{L}\right)$ & $3.27(1.35-6.93)^{\mathrm{a}}$ & $3.71(0.89-23.43)^{\mathrm{a}}$ & $6.41(0.72-34.48)^{b}$ & $3.71(0.72-34.48)$ & $<0.001$ \\
\hline RDW (\%) & $12.9(11.3-17.4)^{\mathrm{a}}$ & $13.7(12-22.5)^{\mathrm{b}}$ & $13.7(12.3-18.1)^{b}$ & $13.5(11.3-22.5)$ & 0.001 \\
\hline Monocyte $\left(\times 10^{3} / \mu \mathrm{L}\right)$ & $0.42(0.14-0.92)$ & $0.37(0.06-1.15)$ & $0.39(0.11-1.46)$ & $0.39(0.06-1.46)$ & 0.128 \\
\hline MPV (fL) & $9.3(7.7-12.4)$ & $9.4(7.2-12)$ & $9.6(7.4-12.2)$ & $9.4(7.2-12.4)$ & 0.091 \\
\hline PDW (\%) & $16.2(14.8-17.1)$ & $16.1(15.3-19.1)$ & $16.3(15.6-17.6)$ & $16.2(14.8-19.1)$ & 0.069 \\
\hline $\mathrm{MCV}(\mathrm{fL})$ & $88.1(57.8-105.6)^{\mathrm{a}}$ & $87(59-101.6)^{\mathrm{ab}}$ & $89.7(78.5-100.8)^{\mathrm{ac}}$ & 87.8 (57.8-105.6) & 0.026 \\
\hline NLR & $2.01(1-9.1)^{a}$ & $3.12(0.55-66.94)^{b}$ & $5.34(1.48-31.56)^{c}$ & $2.80(0.55-66.94)$ & $<0.001$ \\
\hline PLR & $130(66.5-365.8)^{a}$ & $152.3(28.7-771.1)^{b}$ & $167.8(31.6-2568.1)^{b}$ & $141.35(28.7-2568.1)$ & 0.001 \\
\hline$D$-dimer $(\mu \mathrm{g} / \mathrm{L})$ & $315.5(97-1880)^{a}$ & $683.5(67-17700)^{b}$ & $1280(123-7960)^{c}$ & $549(67-17700)$ & $<0.001$ \\
\hline
\end{tabular}

Descriptive statistics are presented as median (minimum-maximum). Pairwise comparisons are shown with "a", "b", "c" symbols. CRP: C-reactive protein; ICU: Intensive care unit; MCV: Mean corpuscular volume; MPV: Mean platelet volume; NLR: Neutrophil-to-lymphocyte ratio; NMR: Neutrophil-to-monocyte ratio; PDW: Platelet distribution width; PLR: Platelet-to-lymphocyte ratio; RDW: Red blood cell distribution width.

Table 3. Univariate and multivariate analysis of patient need for intensive care unit hospitalization

\begin{tabular}{|c|c|c|c|c|}
\hline Variables & \multicolumn{2}{|c|}{ Univariate } & \multicolumn{2}{|c|}{ Multivariate } \\
\hline Age & $1.04(1.02-1.06)$ & 0.001 & $1.02(1.00-1.05)$ & 0.053 \\
\hline Gender (female) & $2.35(1.113-4.95)$ & 0.025 & - & - \\
\hline Lymphocyte & $0.73(0.41-1.30)$ & 0.286 & - & - \\
\hline Neutrophil & $1.28(1.14-1.45)$ & 0.001 & & \\
\hline Monocyte & $3.58(0.70-18.36)$ & 0.126 & - & - \\
\hline Hemoglobin & $0.97(0.95-0.99)$ & 0.001 & & \\
\hline Hematocrit & $0.94(0.88-0.99)$ & 0.038 & - & - \\
\hline MPV & 1.45 (1.04-2.03) & 0.030 & 1.69 (1.07-2.69) & 0.023 \\
\hline NMR & $1.07(1.0-1.10)$ & $<0.001$ & $1.07(1.03-1.12)$ & $<0.001$ \\
\hline Procalcitonin & $7.42(1.68-32.74)$ & 0.008 & - & - \\
\hline CRP & $1.012(1.01-1.02)$ & 0.003 & - & - \\
\hline D-dimer & $1.00(1.00-1.00)$ & 0.020 & - & - \\
\hline
\end{tabular}

CRP: C-reactive protein; MCV: Mean corpuscular volume; MPV: Mean platelet volume; NLR: Neutrophil-to-lymphocyte ratio; NMR: Neutrophil-to-monocyte ratio; PDW: Platelet distribution width; PLR: Platelet-to-lymphocyte ratio; RDW: Red blood cell distribution width.

The patients were followed up throughout their treatment and approximately half of patients in ICU died. Parameters to predict death were evaluated using univariate and multivari- ate analysis. There were significant differences in the neutrophil, NLR, PLR, NMR, procalcitonin, and CRP levels in univariate analysis with values adjusted for age and sex. Multivariable 
Table 4. Receiver operating characteristic curve analysis of parameters to predict the need for intensive care treatment

\begin{tabular}{|c|c|c|c|c|c|c|}
\hline Variables & AUC & $\mathbf{p}$ & $\begin{array}{l}\text { Optimal } \\
\text { threshold }\end{array}$ & Sensitivity & Specifity & Youden \\
\hline Age & 0.695 & $<0.001$ & $>55$ & 83.33 & 54.05 & 0.3739 \\
\hline Hemoglobin & 0.654 & 0.005 & $\leq 112$ & 48.65 & 85.95 & 0.3459 \\
\hline Hematocrit & 0.639 & 0.016 & $\leq 34.8$ & 45.9 & 86.5 & 0.3243 \\
\hline NLR & 0.772 & $<0.001$ & $>4.26$ & 71.4 & 79.5 & 0.5089 \\
\hline PLR & 0.623 & 0.027 & $>145.9$ & 70.3 & 56.8 & 0.2703 \\
\hline NMR & 0.678 & 0.001 & $>17.6$ & 43.24 & 89.19 & 0.3243 \\
\hline Procalcitonin & 0.687 & 0.001 & $>0$ & 59.38 & 71.84 & 0.3121 \\
\hline CRP & 0.727 & $<0.001$ & $>51.9$ & 62.16 & 75.41 & 0.3757 \\
\hline
\end{tabular}

AUC: Area under the curve; CRP: C-reactive protein; MCV: Mean corpuscular volume; NLR: Neutrophil-to-lymphocyte ratio; NMR: Neutrophil-to-monocyte ratio; PDW: Platelet distribution width; PLR: Platelet-to-lymphocyte ratio.

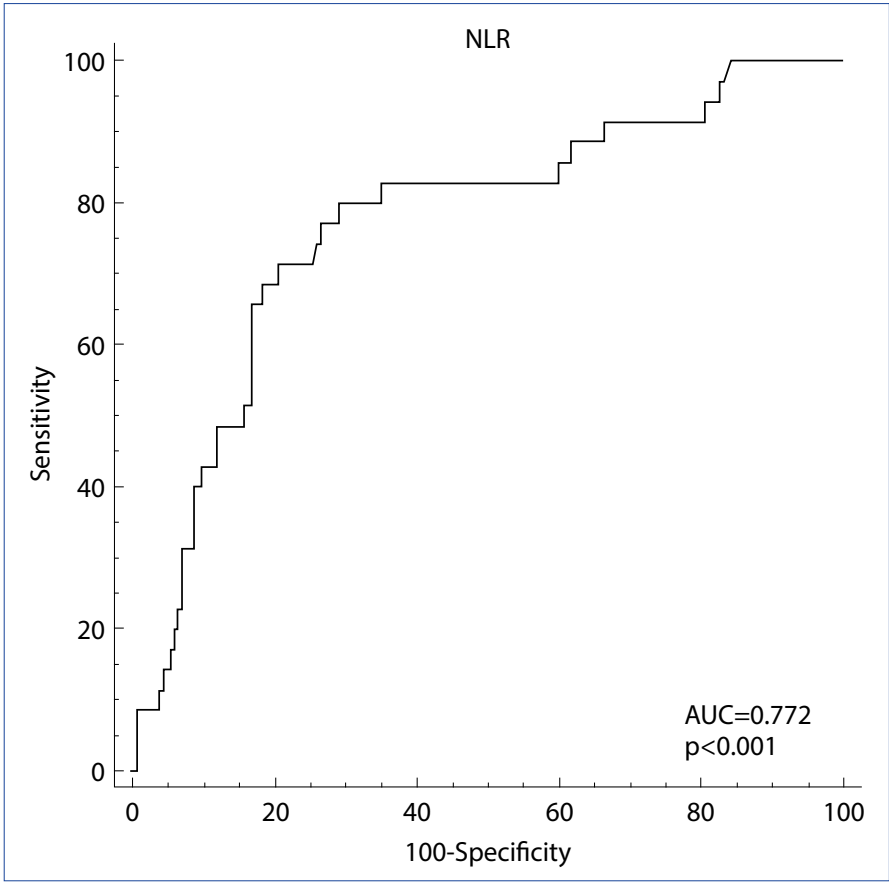

Figure 2. Receiver operating characteristic curve analysis for the neutrophil-to-lymphocyte ratio as a predictor of the need for intensive care unit hospitalization.

AUC: Area under the curve; NLR: Neutrophil-to-lymphocyte ratio.

logistic regression revealed a significant difference in only the NMR (HR: $1.05,95 \% \mathrm{Cl}: 1.03-1.08)$ and procalcitonin (HR: 1.23 , 95\% Cl: 1.03-1.48) values (Table 5).

When the effect of parameters on hospitalization time was examined, it was noted that age, MPV, procalcitonin, CRP, and D-dimer levels $(r=0.429,0.191,0.192,0,259,0,368$, respectively, $\mathrm{p}<0.05$ ) were positively correlated with the length of hospital stay.

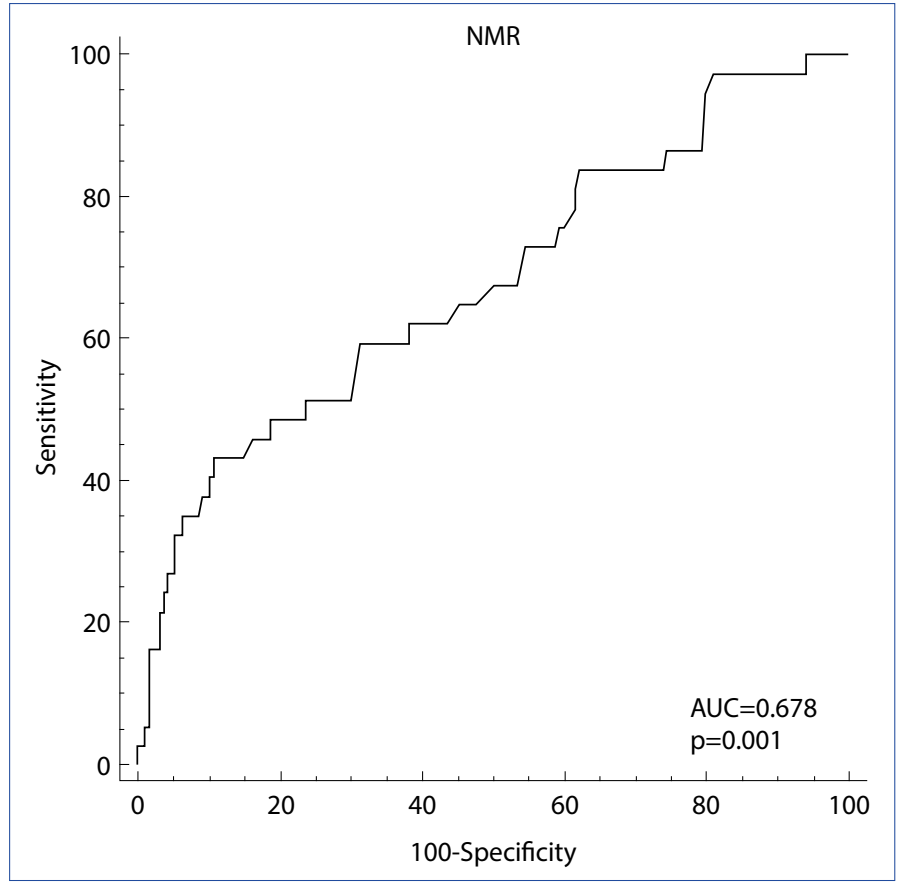

Figure 3. Receiver operating characteristic curve analysis for the neutrophil-to-monocyte ratio as a predictor of the need for intensive care unit hospitalization.

AUC: Area under the curve; NMR: Neutrophil-to-monocyte ratio.

\section{Discussion}

The ability to develop a reliable prognosis at the time of admission will prevent unnecessary hospitalization and help to ensure the optimal use of resources. An early diagnosis and determination of a prognosis is of critical importance in COVID-19 cases. Many studies have been conducted examining the severity of COVID-19; this research was designed to 
Table 5. Univariate and multivariate analysis to predict mortality

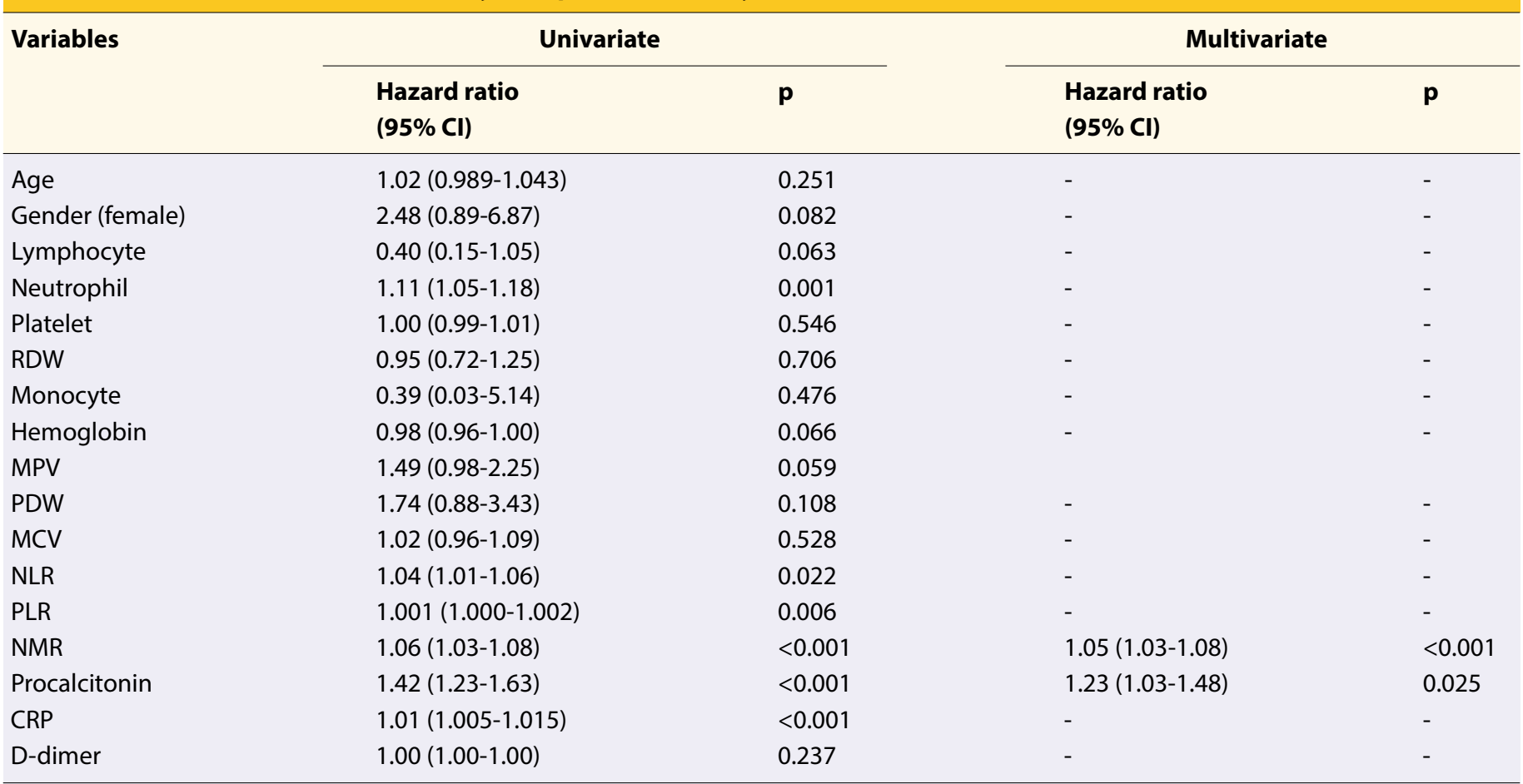

CRP: C-reactive protein; MCV: Mean corpuscular volume; MPV: Mean platelet volume; NLR: Neutrophil-to-lymphocyte ratio; NMR: Neutrophil-to-monocyte ratio; PDW: Platelet distribution width; PLR: Platelet-to-lymphocyte ratio; RDW: Red blood cell distribution width.

help predict both the need for intensive care treatment and patient mortality.

In this study, although the lymphocyte count was significantly lower in the patients who required ICU care and the inpatients $(p=0.003$ and $p<0.001$, respectively), it was not sufficient to predict the need for intensive care or death. Reports in the literature have indicated that a low lymphocyte count, especially a low T lymphocyte count, was very common in ICU patients. There are varied opinions about this change in lymphocyte count, including that it may be a result of an inflammatory cytokine storm, and that SARSCoV-2 infection may interfere with T cells, causing depletion, cell infection, or reduced expansion [10]. Wagner et al. [11] noted that a low lymphocyte percentage was a prognostic marker in COVID-19 patients. Several studies have observed that severe cases presented with a low lymphocyte count, a high neutrophil count, and a high NLR [12]. Liu et al. [13] found in a study of 61 patients that the severity of COVID-19 was associated with the NLR and a cutoff value of 3.13 served to indicate severity. Ciccullo et al. [14] reported that NLR was a useful prognostic factor in the early screening of critical illness. Our findings were consistent with the results of previous studies; the NLR was significantly greater in the ICU patients than the inpatients or outpatients $(p<0.001)$. The NLR was also greater in inpatients than outpatients. The NLR was a significant parameter in predicting the need for intensive care and death. ROC analysis with a cutoff 4.26 indicated that NLR was a valuable predictor of the need for ICU hospitaliza- tion (AUC: 0.77 , sensitivity: 71\%, specificity: 79\%). The most important parameter to predict the need for intensive care treatment was the neutrophil count, with an AUC of 0.782 , sensitivity of $73 \%$, and a specificity of $75 \%$ and a cutoff value of 4.43. Rizo-Téllez et al. [15] found an NMR $>17.75$ to be a good independent risk factor for predicting mortality with a sensitivity of $89.4 \%$ and a specificity of $80 \%$. Peng et al. [16] found that NMR was significantly associated with the severity of COVID-19 (12.4 vs. 8.0 in severe and non-severe patients; $p<0.001$ ). In this study, the NMR value was found to be significant in both univariate and multivariate analysis as a predictor of the need for intensive care. ROC analysis with an NMR cutoff value of 17.6 had an AUC of 0.678 , a sensitivity of $43 \%$, and a specificity of $89 \%$. The NMR was significantly greater in the ICU group in both univariate and multivariate analysis and was a predictor of mortality.

Many studies have suggested that hemoglobin, hematocrit, and RDW values may be independent risk factors associated with severe disease [17]. In a meta-analysis conducted by Lippi et al. [18], RDW was found to be useful for assessing the risk of unfavorable COVID-19 progression. It was reported in another study that an elevated RDW measured at admission and increasing RDW during hospitalization were associated with a significantly higher mortality risk [19]. Similarly, in this study, the RDW and MCV levels were significantly lower and the hemoglobin and hematocrit values were significantly higher in outpatients compared with the ICU and inpatient groups. With values adjusted for age and sex, univariate analysis re- 
vealed lower hemoglobin and hematocrit values in the ICU group. However, the RDW was not significant in univariate or multivariate analysis of patient need for ICU care or as a predictor of mortality, nor did this parameter correlate with the length of hospitalization.

It was observed in a meta-analysis that evaluated 1779 patients that a low platelet count was associated with increased risk of severe disease and mortality [20,21]. Furthermore, the MPV and PDW were higher in non-survivors on admission day in another study [8]. The MPV can provide important information about the course and prognosis in many pathological conditions [22]. This includes diseases such as cardiovascular diseases, respiratory diseases, Crohn's disease, rheumatoid arthritis, inflammatory disease such as juvenile systemic lupus erythematous, diabetes mellitus, and many neoplastic diseases [23-28]. It has been demonstrated that inflammatory cytokines regulate both prothrombotic and proinflammatory events by regulating thrombopoiesis and MPV. A high MPV is associated with cardio and cerebrovascular disorders and low-grade inflammatory conditions prone to arterial and venous thrombosis $[29,30]$. It is thought that a high MPV, which is also associated with hypercoagulation and inflammation, may be associated with COVID-19 complications. The intensity of systemic inflammation is also correlated with different sized platelets [29]. In this study, there was no significant difference between groups in terms of platelets, MPV or PDW. However, univariate analysis indicated that the MPV and PDW were significant, while multivariate analysis yielded only the MPV as a significant predictor of the need for intensive care treatment. These parameters were not associated with mortality prediction or the length of hospitalization.

Wang [31] reported that the CRP level was an early parameter that indicated disease severity, and Yao et al. [32] noted that the D-dimer level correlated with disease severity and was a reliable prognostic parameter for in-hospital mortality. In a study of 4103 patients, it was found that age and the CRP and D-dimer levels were the strongest risk factors affecting hospitalization [33]. Tang et al. [34] found that an elevated D-dimer level was common in COVID-19 deaths, and Wang et al. [35] developed a laboratory model including age, neutrophil and lymphocyte counts, and CRP and D-dimer levels. We found that procalcitonin, CRP, and D-dimer values were related to disease severity. Procalcitonin, CRP, and D-dimer values were significantly higher in the ICU patients than the inpatients or outpatients $(p<0.001)$. The procalcitonin, CRP, and D-dimer levels in inpatients were also higher than in the outpatients. They were significantly higher in the ICU patients than in the outpatients or the inpatients. They also were significant in univariate analysis to predict the need for intensive care. A D-dimer level $>1000(\mu \mathrm{g} / \mathrm{L})$ and a CRP level $>10$ times the normal level are among the hospitalization criteria in the adult patient treatment guideline of the Turkish Ministry of Health. In this study, similar to other reports in the literature, age and CRP and D-dimer levels were observed to be the parameters most associated with the duration of hospitalization $(r=0.429,0.259,0.368$, respectively; $p<0.01)$. The lymphocyte count was not associated with the duration of hospitalization.

\section{Conclusion}

There are many opinions in the literature about laboratory parameters and the prediction of mortality and severity in this new disease. Thus far, the most valuable parameter to predict the need for intensive care is the neutrophil count (AUC, sensitivity, and specificity of $0.782,73 \%$, and $75 \%$, respectively), and the best predictive marker of mortality is the NMR and the procalcitonin value. Age, the MPV, and the procalcitonin, CRP, and D-dimer levels were found to be positively correlated with the length of hospital stay.

Acknowledgements: The authors thank all of the laboratory staff for their contributions to this research.

Conflict of Interest: The authors declare that they have no conflict of interest.

Ethics Committee Approval: This study was approved by the Health Sciences University Sisli Hamidiye Etfal Training and Research Hospital Clinical Research Ethics Committee (Date: 22.09.2020; No: 3002).

Financial Disclosure: There are no financial conflicts of interest to disclose.

Peer-review: Externally peer-reviewed.

Authorship Contributions: Concept - M.S.O.; Design - M.S.O.; Supervision - E.S.; Funding - None; Materials - I.S.; Data collection \&/or processing - A.A., P.Y.; Analysis and/or interpretation - G.O.; Literature search - M.S.O.; Writing - M.S.O.; Critical review - E.S.

\section{References}

1. WHO Coronavirus Disease (COVID-19) Dashboard. Available at: https://covid19.who.int/. Accessed Mar 16, 2021.

2. Zheng J. SARS-coV-2: an emerging coronavirus that causes a global threat. Int J Biol Sci 2020;16(10):1678-85. [CrossRef]

3. Petersen E, Koopmans M, Go U, Hamer DM, Petrosillo N, Castelli $F$, et al. Comparing SARS-CoV-2 with SARS-CoV and influenza pandemics. Lancet Infect Dis 2020;20:e238-44. [CrossRef]

4. Vekaria B, Overton C, Wisniowski A, Ahmad S, Aparicio-Castro A, Curran-Sebastian J, et al. Hospital length of stay for COVID-19 patients: data-driven methods for forward planning. BMC Infect Dis. 2020 Oct 1. doi: 10.21203/rs.3.rs-56855/ v1. [Epub ahead of print]. [CrossRef]

5. Lippi G, Wong J, Henry BM. Hypertension in patients with coronavirus disease 2019 (COVID-19): a pooled analysis. Pol Arch Intern Med 2020;130(4):304-9. [CrossRef]

6. Lippi G, Plebani M. The critical role of laboratory medicine during coronavirus disease 2019 (COVID-19) and other viral outbreaks. Clin Chem Lab Med 2020;58(7):1063-9. [CrossRef] 
7. Yuan X, Huang W, Ye B, Chen C, Huang R, Wu F, et al. Changes of hematological and immunological parameters in COVID-19 patients. Int J Hematol 2020;112(4):553-9. [CrossRef]

8. Yun H, Sun Z, Wu J, Tang A, Hu M, Xiang Z. Laboratory data analysis of novel coronavirus (COVID-19) screening in 2510 patients. Clin Chim Acta 2020;507:94-7. [CrossRef]

9. T.C. Sağlık Bakanlığı Halk Sağlığı Genel Müdürlüğü. Covid-19 (Sars-Cov-2 enfeksiyonu) erişkin hasta tedavisi 2020. Available at: https://covid19.saglik.gov.tr/TR-66301/covid-19-rehberi. html. Accessed Mar 16, 2021.

10. Tavakolpour S, Rakhshandehroo T, Wei EX, Rashidian M. Lymphopenia during the COVID-19 infection: What it shows and what can be learned. Immunol Lett 2020;225:31-2. [CrossRef]

11. Wagner J, DuPont A, Larson S, Cash B, Farooq A. Absolute lymphocyte count is a prognostic marker in Covid-19: A retrospective cohort review. Int J Lab Hematol 2020;42(6):761-5.

12. Kong M, Zhang H, Cao X, Mao X, Lu Z. Higher level of neutrophil-to-lymphocyte is associated with severe COVID-19. Epidemiol Infect 2020;148:e139. [CrossRef]

13. Liu J, Liu Y, Xiang P, Pu L, Xiong H, Li C, et al. Neutrophil-to-lymphocyte ratio predicts critical illness patients with 2019 coronavirus disease in the early stage. J Transl Med 2020;18(1):206.

14. Ciccullo A, Borghetti A, Zileri Dal Verme L, Tosoni A, Lombardi F, Garcovich M, et al; Gemelli Against COVID Group. Neutrophil-to-lymphocyte ratio and clinical outcome in COVID-19: a report from the Italian front line. Int J Antimicrob Agents 2020;56(2):106017. [CrossRef]

15. Rizo-Téllez SA, Méndez-García LA, Flores-Rebollo C, AlbaFlores F, Alcántara-Suárez R, Manjarrez-Reyna AN, et al. The neutrophil-to-monocyte ratio and lymphocyte-to-neutrophil ratio at admission predict in-hospital mortality in Mexican patients with Severe SARS-CoV-2 infection (Covid-19). Microorganisms $2020 ; 8(10): 1560$ [CrossRef]

16. Peng F, Lei S, Wu C, Yu B, Zhong Y, Wu S. Neutrophil percentage and neutrophil-to-monocyte ratio as independent risk factors in the severity of COVID-19. Research Square. 2020 Sep 23. doi:10.21203/rs.3.rs-52622/v1. [Epub ahead of print]. [CrossRef]

17. Tao Z, Xu J, Chen W, Yang Z, Xu X, Liu L, et al. Anemia is associated with severe illness in COVID-19: A retrospective cohort study. J Med Virol 2021;93(3):1478-88. [CrossRef]

18. Lippi G, Henry BM, Sanchis-Gomar F. Red blood cell distribution is a significant predictor of severe illness in coronavirus disease 2019. Acta Haematol. 2020 Aug 25. doi: 10.1159/000510914. [Epub ahead of print]. [CrossRef]

19. Foy BH, Carlson JCT, Reinertsen E, Padros I Valls R, Pallares Lopez $R$, Palanques-Tost $E$, et al. Association of red blood cell distribution width with mortality risk in hospitalized adults with SARS-CoV-2 infection. JAMA Netw Open 2020;3(9):e2022058.

20. Lippi G, Salvagno G, Montagnana M, Lima-Oliveira G, Guidi G, Favaloro E. Quality standards for sample collection in coagulation testing. Semin Thromb Hemost 2012;38(6):565-75. [CrossRef]
21. Bao C, Tao X, Cui W, Yi B, Pan T, Young KH, et al. SARS-CoV-2 induced thrombocytopenia as an important biomarker significantly correlated with abnormal coagulation function, increased intravascular blood clot risk and mortality in COVID-19 patients. Exp Hematol Oncol 2020;9:16. [CrossRef]

22. Korniluk A, Koper-Lenkiewicz OM, Kamińska J, Kemona H, Dymicka-Piekarska V. Mean platelet volume (MPV): new perspectives for an old marker in the course and prognosis of inflammatory conditions. Mediators Inflamm 2019;2019:9213074.

23. Slavka G, Perkmann T, Haslacher H, Greisenegger S, Marsik C, Wagner OF, et al. Mean platelet volume may represent a predictive parameter for overall vascular mortality and ischemic heart disease. Arterioscler Thromb Vasc Biol 2011;31(5):1215-8.

24. Shah B, Oberweis B, Tummala L, Amoroso NS, Lobach I, Sedlis $\mathrm{SP}$, et al. Mean platelet volume and long-term mortality in patients undergoing percutaneous coronary intervention. Am J Cardiol 2013;111(2):185-9. [CrossRef]

25. Liu S, Ren J, Han G, Wang G, Gu G, Xia Q, et al. Mean platelet volume: a controversial marker of disease activity in Crohn's disease. Eur J Med Res 2012;17(1):27. [CrossRef]

26. Gasparyan AY, Sandoo A, Stavropoulos-Kalinoglou A, Kitas GD. Mean platelet volume in patients with rheumatoid arthritis: the effect of anti-TNF-a therapy. Rheumatol Int 2010;30(8):1125-9.

27. Yavuz S, Ece A. Mean platelet volume as an indicator of disease activity in juvenile SLE. Clin Rheumatol 2014;33(5):637-41.

28. Li N, Yu Z, Zhang X, Liu T, Sun YX, Wang RT, et al. Elevated mean platelet volume predicts poor prognosis in colorectal cancer. Sci Rep 2017;7(1):10261. [CrossRef]

29. Gasparyan AY, Ayvazyan L, Mikhailidis DP, Kitas GD. Mean platelet volume: a link between thrombosis and inflammation? Curr Pharm Des 2011;17(1):47-58. [CrossRef]

30. Yolcu S, Beceren GN, Tomruk Ö, Doguç DK, Balbaloglu O. Can mean platelet volume levels of trauma patients predict severity of trauma? Platelets 2014;25(4):279-84. [CrossRef]

31. Wang L. C-reactive protein levels in the early stage of COVID-19. Med Mal Infect 2020;50(4):332-4. [CrossRef]

32. Yao Y, Cao J, Wang Q, Shi Q, Liu K, Luo Z, et al. D-dimer as a biomarker for disease severity and mortality in COVID-19 patients: a case control study. J Intensive Care 2020;8:49. [CrossRef]

33. Petrilli CM, Jones SA, Yang J, Rajagopalan $H$, O'Donnell $L$, Chernyak $Y$, et al. Factors associated with hospital admission and critical illness among 5279 people with coronavirus disease 2019 in New York City: prospective cohort study. BMJ 2020;369:m1966. [CrossRef]

34. Tang N, Li D, Wang X, Sun Z. Abnormal coagulation parameters are associated with poor prognosis in patients with novel coronavirus pneumonia. J Thromb Haemost 2020;18(4):844-7.

35. Wang K, Zuo P, Liu Y, Zhang M, Zhao X, Xie S, et al. Clinical and laboratory predictors of in-hospital mortality in patients with coronavirus disease-2019: a cohort study in Wuhan, China. Clin Infect Dis 2020;71(16):2079-88. [CrossRef] 